\title{
A Long-Term Human-Robot Proxemic Study
}

\author{
Michael L. Walters, Mohammedreza A. Oskoei, Dag Sverre Syrdal and \\ Kerstin Dautenhahn, Member, IEEE
}

\begin{abstract}
A long-term Human-Robot Proxemic (HRP) study was performed using a newly developed Autonomous Proxemic System (APS) for a robot to measure and control the approach distances to the human participants. The main findings were that most HRP adaptation occurred in the first two interaction sessions, and for the remaining four weeks, approach distance preferences remained relatively steady, apart from some short periods of increased distances for some participants. There were indications that these were associated with episodes where the robot malfunctioned, so this raises the possibility of users trust in the robot affecting HRP distance. The study also found that approach distances for humans approaching the robot and the robot approaching the human were comparable, though there were indications that humans preferred to approach the robot more closely than they allowed the robot to approach them in a physically restricted area. Two participants left the study prematurely, stating they were bored with the repetitive experimental procedures. This highlights issues related to the often incompatible demands of keeping experimental controlled conditions vs. having realistic, engaging and varied HRI trial scenarios.
\end{abstract}

\section{INTRODUCTION}

$\mathrm{D}$ omestic and service robots should not just be technically capable, but also be able to take account of human factors in their working environment. An important area of research especially relevant to mobile robots, is Human-Robot Proxemics (HRP). Between humans, the research field of Proxemics investigates how humans negotiate, manipulate and use interpersonal distances as a means of social communication (cf. Hall [1][2], Sommer[3] and Burgoon \& Jones [4]). Due to the physical presence of robots, it has been shown that people respond to them socially in some ways similarly to how they respond to other humans or pets. Breazeal [5] found that humans responded to expressive zoo-morphic robots with regard to turn-taking in speech communication and also respected the robot's inter-personal space. Nomura et al. [6] found that both participants' negative attitudes and anxiety towards a small size humanoid robot, RobovieM (29 $\mathrm{cm}$ tall and $1.9 \mathrm{~kg}$ ), had statistically significant effects on users preferred (comfortable) robot approach distances. Hüttenrauch et al. [7] found that most HRI user trial participants kept interpersonal distances from the robot corresponding to Hall's Personal Spatial Zone $(0.45 \mathrm{~m}$ to $1.2 \mathrm{~m})$. In initial HRI proxemic trials we also found that groups of children tended

Manuscript received June $1^{\text {st }} 2011$. This work was supported by the LIREC (LIving with Robots and intEractional Companions) Project, funded by the European Commission under Contract number FP7-215554.

Michael L Walters ( M.L.Walters@herts.ac.uk), Dag Sverre Syrdal (D.S.Syrdal@herts.ac.uk), Mohammedreza A. Oskoei (M.A.Oskoei @herts.ac.uk) and Kerstin Dautenhahn (K.Dautenhahn@herts.ac.uk) are all with the Adaptive Systems Research Group at the University of Hertfordshire. to approach a PeopleBot ${ }^{\mathrm{TM}}$ robot to similar distances on first encounter [8], but for individual adults approaching the same robot, the approach distances were more ambivalent and inconclusive [9][10]. A different HRI experiment used a similar mechanoid appearance PeopleBot robot, fitted with a simple pointing arm and which used different voice styles. Participants initially encountering the robot took significantly different comfortable approach distances [11] It was suggested that these differences may be caused by initial uncertainty due to perceived inconsistencies between the robots appearance and voice styles.

More recently, a number of Human-Robot Interaction studies have identified more factors which affect HRP distances. Koay et al. [12] found that participants generally allowed robots to approach more closely during physical interactions (e.g. taking and receiving objects) than under verbal (issuing commands e.g. "turn left", "forward" etc.) or no interaction conditions (e.g the robot passes through the same room). Although people generally preferred more humanoid appearance robots to keep a further distance away than mechanoid robots, the height of a robot (short = $1.2 \mathrm{~m}$, tall $=1.4 \mathrm{~m}$ ) had no significant effect on HRP [13] [14]. Interestingly however, we found that users' preferences for particular robot attributes, including appearance and height, did affect their HRP preferred distances with regard to all robot types [15]. There were also indications that participants preferences and HRP distance preferences were also affected by their personality factors (mainly the extraversion/intro-version factor from the "big five" Personality Inventory [16]). Takayama and Pantofaru [17] have also found that robot head shape, the orientation of the interacting robot, the gender and personality of the user all have significant effects on HRP. Mumm and Mutlu [18] found that different gaze behaviours of a humanoid (Wakamuru [19]) robot affected peoples preferred interaction distance, but also that this was affected by their degree of liking for the robot. These findings indicate that HRP exhibits at least some of the richness that has been observed in human-human proxemics. For both human-human proxemics and HRP, significant differences in proxemic distances are typically of the order of $2 \mathrm{~cm}$ to 10 $\mathrm{cm}$. It seems that many of these significant differences are relatively small and that humans would not perceive them (consciously) in absolute terms, but as comparative differences or changes during HRIs. Therefore, we suggest that relatively small dynamic changes in proximity which occur during HRIs are an important area for investigation. However, most findings for HRP to date were gained in HRI studies which used remotely controlled or semi-autonomous robot control techniques in resource intensive HRI trials [20]. The HRP distance measurements were made either by 
hand, or by using relatively course measurement techniques. In order to confirm and extend these findings, investigate whether other factors might apply to HRP interactions, and also effectively measure and quantify any effects, it is necessary to use "on-board" autonomous robot HRP sensing and control capabilities. In this way, it would be possible to measure and record dynamic HRP interactions continuously and accurately, and in conjunction with other established means of data collection (video, questionnaires, interviews etc.) provide for a more complete analyses of the interactions. In order for a robot to regulate proxemic distances to users autonomously, we have developed an Autonomous Proxemic System (APS) which has been tested and evaluated under semi-realistic conditons [21]. We have gone on to perform a Long-Term HRI study using the APS to address the following key research questions: 1) If, and in what way, participants' HRP preferences changed in longterm experiments, 2) If, and how, those preferences differed in two different contexts (relatively open and constrained spaces), and 3) Do differences of HRP preferences exist between human-to-robot and robot-to-human approaches. Based on findings from our previous studies [11][12][22] we expected that: 1) Participants HRP preferences would reduce during initial HRI sessions, then stabilise over the remaining part of the study. 2) That they would allow the robot to approach closer in the open area than in the confined area, and 3) They would tend to approach the robot more closely than they would prefer the robot to approach themselves.

The following section briefly outlines the APS which allows a mobile robot to provide on-board measurement and control of HRP distances. The latter sections present a Long-Term HRP study which served two main purposes: Firstly to evaluate the performance of the APS under extended HRI trial conditions. Secondly to track changes in users HRP distance preferences over a six week period. This study covered the same general ground as one of our previous HRP studies [12], but took advantage of the increased accuracy and efficiency of the APS data collection capabilities to provide more refined data collection and analyses.

\section{AutONOMOUS PROXEMIC SYSTEM}

The Autonomous Proxemic System (APS) for our robot companion has been developed to automatically measure and control for a given desired HRP distance. The APS can locate and track people within the robot's immediate environment, using computer vision techniques to detect either a face or upper body of a person within its camera range. It also applies a leg detection algorithm to laser range finder data (cf. Hassch et al. [23], Zheng and Meng [24], Zender et al. [25], Bellotto \& $\mathrm{Hu}$ [26]). In addition, infra-red (IR) and sonar sensors are also used to perceive and track human motions and obstacles. The system applies a sensor fusion technique to discriminate humans from objects. Previously, tests of the system have found that the APS measures HRP distance to within an acceptable error margin of approximately $\pm 1.5 \%$, with a repeatability of approximately $2 \%$. This equates to an error of approximately $2 \mathrm{cms}$ within the range of distances specified for Hall's personal zone [27]. More complete details are available in Oskoei et al. 2010 [21].

\section{EXPERIMENTAL SETUP AND PROCEDURE}

The study was performed in the University of Hertfordshire "Robot House" which is a typical residential house located off-campus that has been fitted out specially for running HRI user trials in a neutral and ecologically sound setting for HRI studies. From participants own perspectives, HRI trials in the Robot House are more similar to visiting an acquaintance's or friend's home. This setting clearly differs from a lab based study, which would require a more formidable entry through the University reception areas, and then trying make participants feel relaxed in a relatively artificial and non-domestic laboratory or office environment.

Seven participants were recruited who were mainly mature and retired, which is relatively unusual for University based HRI trials which often tend to dominantly recruit students, academic and support staff. The mature participant sample were specifically targeted for this study, as we envision that one of the most immediate useful areas for domestic robots will be in care of the elderly and people with special needs. This is an area where some (technically) relatively simple robot functionalities, such as fetch, follow and carry tasks, and cognitive prosthetic (i.e. reminder) tasks, could make a difference between someone having to go into care or staying in their own home. Participants were informed that they could withdraw from the experiment at any point if they wished. Also note that the participants had to travel themselves to the Robot House. Thus, attending the experiment involved a non-trivial personal commitment from the participants.

There were 3 male and 4 female participants. Most participants ages ranged from late $50 \mathrm{~s}$ to early $70 \mathrm{~s}$, with one female participant being in her mid-forties and one male participant in his mid-twenties. As a group, they were relatively experienced in the use of computers for personal or work purposes. One participant (NG1), left the study after week 5 due to a family emergency and two participants (FU1 and UN1) left the study, stating they found the

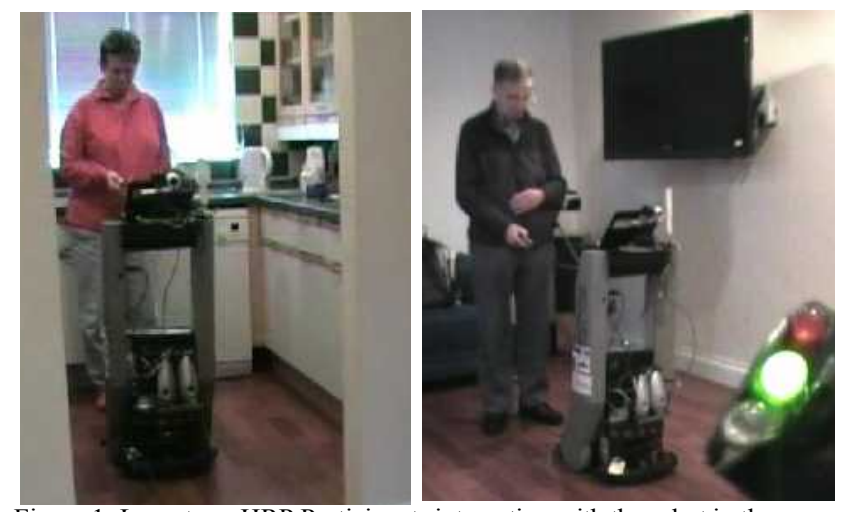

Figure 1: Long-term HRP Participants interacting with the robot in the Kitchen area (left) and Living room area (Right) 
repetitive experimental procedure "boring" and they had hoped for more varied and interesting interactions with the robot.

A PeopleBot ${ }^{\mathrm{TM}}$ [28] robot, running the APS, was used for the study. Before the first trial, participants signed consent forms and completed personality and demographic questionnaires. The experimental procedure and purpose was explained and they first had a short practice with a wireless remote control which they used to set their preferred robot approach distance (Figure 2). This remote control has three buttons - Forward, Backward and Set and is linked by radio to the robot and APS. The Forward and Backward buttons are used by each participant to adjust the robots position until they are satisfied. They then press the Set control button to trigger the robot to record the actual measured distance from the APS.

Each trial session involved the robot approaching each participant in two areas: a relatively open area in the centre of the main Living room, and a smaller, enclosed Kitchen area. (see Figure 1). These particular areas were chosen as in our previous HRI studies [29], participants had reported that they found approaches by the robot to be less comfortable when they were backed up against a wall or restricted from moving backwards, compared to robot approaches in open areas.

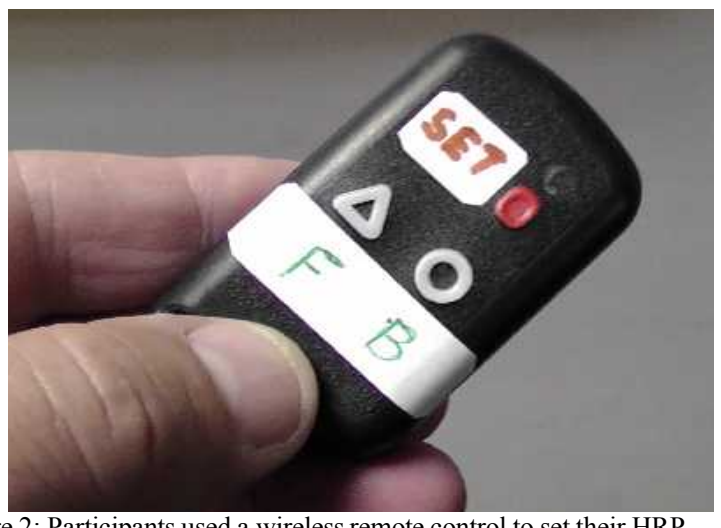

Figure 2: Participants used a wireless remote control to set their HRP preferences. Note: $\mathrm{F}=$ Forward, $\mathrm{B}=$ Backward, $\mathrm{SET}=$ Set $/$ Record HR distance.

Each session consisted of the same basic procedure and was carried out for each participant approximately once a week (according to their availability): The robot approached each participant to a standard $60 \mathrm{~cm}$ distance in the first area (either the kitchen or living room). The participant then adjusted the robots position (backwards or forwards) using the remote control, and their preferred Robot to Human (RH) distance was recorded automatically when the Set button was pressed. This was repeated three times to ensure a consistent measurement. The participant then approached the stationary robot three times. Each time they pressed the Set button at their preferred distance to the robot. This distance was also recorded by the APS. The whole procedure was then repeated for the other area. In successive weeks, the order of area presentation was reversed, so in week one, if they performed the trial first in the Kitchen, then the living room, the following week, they would perform the trial run in the Living room first, then the kitchen. Each trial run was recorded on video, and after both trial runs in the two areas were completed, a short standard post trial questionnaire was completed. After the post trial questionnaire, a de-brief interview was then performed, typically about 15-20 minutes long, where their perceptions and opinions could be explored in detail.

\section{RESUlts}

\section{A. Results from the Long-term Proxemics Study - An Overview:}

The results are consistent with our previous findings [12] that the main changes in users' HRP preferences occur between the first and second interactions with a robot, after which proxemic preferences tend to stabilise. Interestingly, this effect is primarily seen in those participants who first interacted with the robot in the kitchen, suggesting that it is not necessarily a function of the novelty of the robot, but the context of the experimental situation as a whole. Also, idiosyncratic factors, both in the individual's perceptions of the robot, the context (both in terms of task and situational factors) and specific responses to these appear to play a role.

\section{B. Descriptives of Proxemic Preferences}

TABLE 1 CHANGES IN PREFERENCES FOR PARTICIPANTS WITH FIRST INTERACTION IN THE KITCHEN(M)

\begin{tabular}{lcccccc}
\hline Participant & RHKIW1 & RHKIW2 & RHKIW3 & RHKIW4 & RHKIW5 & RHKIW6 \\
\hline \hline DT1 & 0.56 & 0.53 & 0.53 & 0.57 & 0.54 & 0.49 \\
FU1 & 0.39 & 0.29 & & & & \\
TG1 & 0.68 & 0.52 & 0.57 & 0.53 & 0.57 & 0.56 \\
UN1 & 0.6 & 0.48 & 0.43 & 0.29 & & \\
\hline
\end{tabular}

TABle 2 Changes IN PREFERENCES FOR PARTICIPANTS WITH FIRST INTERACTION IN THE LIVING ROOM(M)

\begin{tabular}{|c|c|c|c|c|c|c|}
\hline Participant & RHLRW1 & RHLRW2 & RHLRW3 & RHLRW4 & RHLRW5 & RHLRW6 \\
\hline KT1 & 0.48 & 0.49 & 0.52 & 0.5 & 0.45 & 0.49 \\
\hline NG1 & 0.32 & 0.31 & 0.3 & 0.3 & 0.29 & \\
\hline TG2 & 0.47 & 0.39 & 0.43 & 0.48 & 0.44 & 0.49 \\
\hline
\end{tabular}

Key: $\mathrm{RH}=$ Robot to Human approach, $\mathrm{KI}=$ in Kitchen, LR = in Living Room, $\mathrm{Wn}=$ Week $\mathrm{n}$.

Tables 1-2 and Figures 3-4 illustrate the changes in proxemic preferences in the initial room as exhibited by the participants. An interesting observation is that the participants who had their first interaction in the kitchen has a more marked decrease in preferred distance between Week 1 and 2. Also, it seems that, with one exception (UN1), preferences stabilise after week 2. Participant UN1's responses seem to be anomalous. However, the interviews with UN1 suggest that this might be due to an artefact of how the sensors responded to his behaviour as this exchange from session 3 suggests:

UN1: "the machine tended to respond to my movement...until someone pointed out that it was reacting to my hand, so I had to make sure that my hand wasn't in view of the sensors...This time I deliberately lifted it to avoid the sensor reacting". 


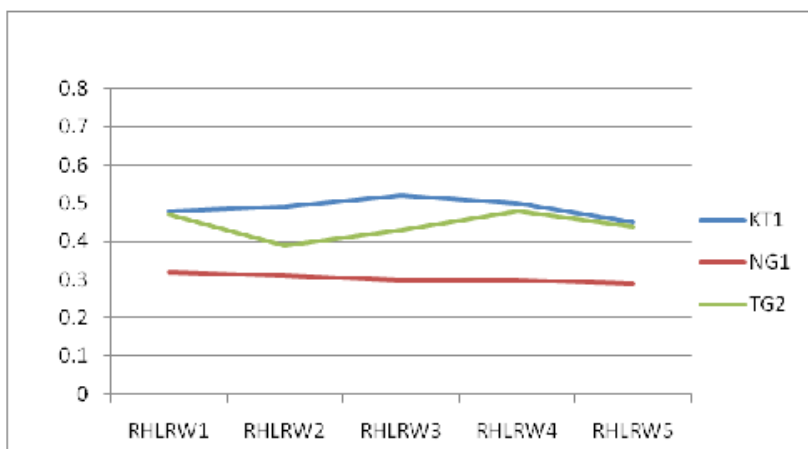

Figure 3 Changes in Robot to Human Approach distance (m) Preferences for Participants with first interaction in the Living Room

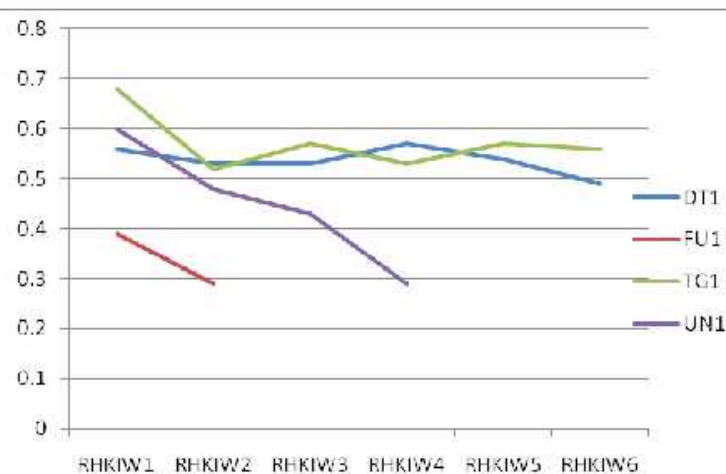

ligure 4 Changes in Robot to Iluman Approach Distances (m) Preferences for Participants with first interaction in the Kitchen

This suggests that such changes mainly come from participants gradually getting to grips with how the robot sensors worked and using that knowledge to make the robot come closer.

C. Overall Mean Difference between Robot to Human and Human to Robot approaches

TABLE 3 DifFERENCES BETWEEN HUMAN TO ROBOT AND RoBOT TO HUMAN APPROACH DISTANCES (M), FOR DIFFERENT LOCATIONS AND

\begin{tabular}{lllllll}
\hline Place & Week1 & Week2 & Week 3 & Week 4 & Week 5 & Week 6 \\
\hline \hline Kitchen & 0.04 & 0.06 & 0.06 & 0.03 & 0.08 & 0.01 \\
Living Rom & 0.04 & 0.04 & 0.03 & 0.05 & 0.02 & -0.01 \\
\hline
\end{tabular}

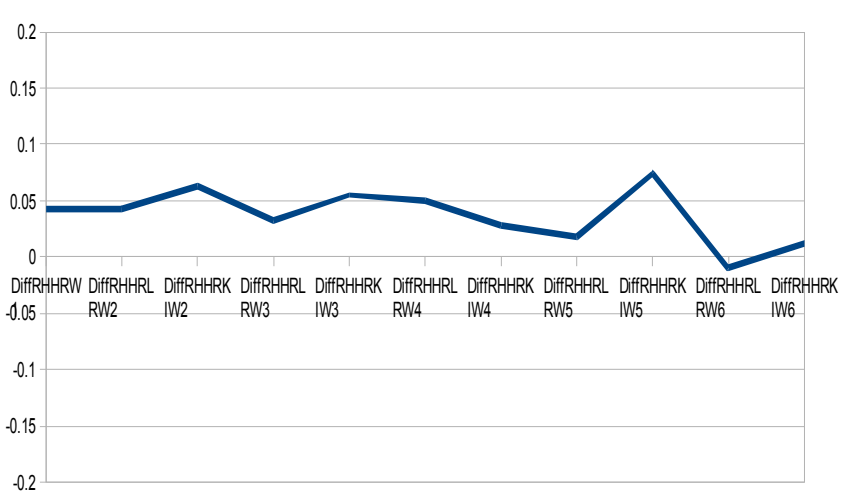

Figure 5: Overall Mean Difference $(\mathrm{m})$ between Robot to Human and Human to Robot Approaches by Session
Table 3 and Figure 5 indicate that overall, participants approached the robot to about $0.05 \mathrm{~m}(5 \mathrm{~cm})$ closer than they allowed the robot to approach them. The main deviation from this was in week 5 for the interactions that took place in the kitchen. Examining the participants' responses and the video record from this session, there seemed to be an issue with the robot's behaviour in this week. Participants highlighted that the robot seemed different in the interactions taking place in the kitchen.

DT1: "It seemed to be less well-oriented today...it seemed a bit faulty today."

KT1: "I thought it whirred more loudly today, but it could have been just my imagination...it came in a bit crooked on the approach this week...It seemed less of a problem in the living room."

NG1: "It moved fairly purposefully until it got there, but before I pressed anything it seemed to wander forwards and backwards a bit... It hadn't done this before... It might have done this before, but I hadn't noticed."

TABLE 4 DIFFERENCE BETWEEN HRP IN LIVING ROOM AND KITCHEN FOR PARTICIPANTS WHOSE FIRST INTERACTION WAS IN THE KITCHEN

\begin{tabular}{llllll}
\hline Participant & Week 2 & Week 3 & Week 4 & Week 5 & Week 6 \\
\hline \hline DT1 & 0 & -0.02 & -0.02 & 0.03 & 0.06 \\
FU1 & 0.11 & & & & \\
TG1 & 0 & -0.05 & 0.02 & 0.16 & 0.01 \\
UN1 & 0.07 & -0.04 & 0.06 & & \\
\hline \hline
\end{tabular}

TABLE 5 DIFFERENCE BETWEEN HRP IN LIVING ROOM AND KITCHEN FOR PARTICIPANTS WHOSE FIRST INTERACTION WAS IN THE LIVING ROOM

\begin{tabular}{llllll}
\hline Participant & Week 2 & Week 3 & Week 4 & Week 5 & Week 6 \\
\hline \hline KT1 & -0.01 & 0.1 & -0.02 & 0.04 & -0.02 \\
NG1 & -0.02 & -0.01 & -0.05 & 0.01 & \\
TG2 & -0.01 & -0.01 & 0.13 & 0.1 & 0.07 \\
\hline \hline
\end{tabular}

Tables 4-5 and Figures 6-7 suggest that the differences (with some exceptions) were small between the participants' proxemic preferences for each room. This is despite the fact that participants, tended to express a strong difference in felt preferences, this could be due to the tasks they envisaged doing with the robot:

TG2: "In the kitchen I wanted it to come closer...because the room is smaller...or maybe that in the kitchen I was thinking more about putting things on the tray. In the living room, I was judging it as if I was going to do something with it."

$\mathrm{UN1}$ : "I felt that when we were working in the kitchen that it was a much more real situation...I'd be in the kitchen, I would want the robot to come closer so I could put something on its tray"

Another reason was the perceived amount of space available to the participants in the different rooms:

NG1:I mean, it was a lot of space on each side in the living room and I suppose I felt it was closer in the kitchen.

KT1:"[In the Kitchen]...I was aware of standing with my back to something, not in the middle of a free room. So I would rather have more room before me as I lent forward." 


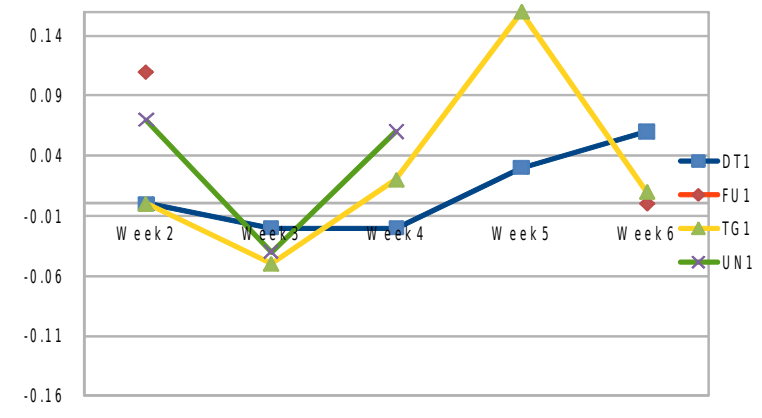

Figure 6 Difference between proxemic distances in the Living room and Kitchen for participants whose first interaction was in the Kitchen (m)

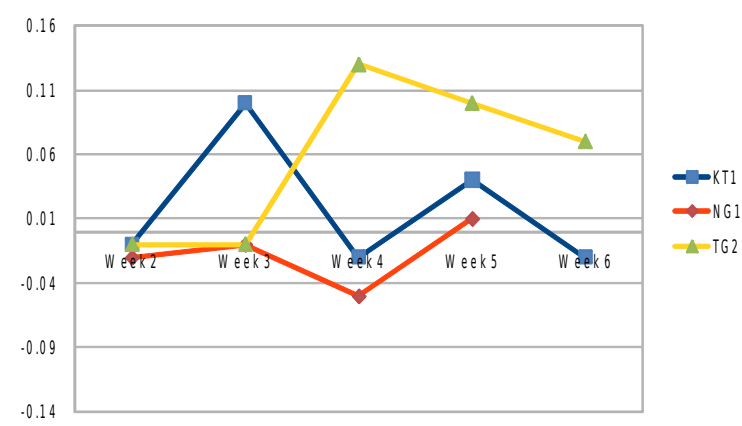

Figure 7 Difference between proxemic distances in the living room and kitchen for participants whose first interaction was in the Living Room

Note that these statements from Week 2 do not necessarily correspond to the observed distances between the participants and the robot.

\section{DISCUSSION}

Participants HRP preferences stabilised within the first two sessions in line with findings from our previous studies [11][12][22]. However, it is not clear generally if participants' acclimatisation happens during their first contact session, or after a period of reflection and then becomes apparent in the second session. The enhanced functionality of the APS provides an opportunity to track how HRP acclimatisation happens in real-time in future work.

Although not statistically significant, primarily due to the low numbers of participants, there were indications that during sessions when the robot malfunctioned, participants HRP distance preferences increased by some small amount. It could be argued that distances would in fact reflect (participants perceptions of) safety distances. However, during the post-trial interviews, safety issues were only raised once, and this was not linked with a robot malfunction episode. However, even when the robot did collide with a participant, the actual safety hazard is (perceived as) minimal and therefore the robot was not perceived as particularly threatening. This would explain the relatively small differences in HRP measures, and also the lack of any mention of these episodes in the interviews.
Although participants consciously appeared not to notice, the HRP record shows that there was an effect and this may indicate a link between users' trust in the robot and HRP distance.

Our previous studies [8], found a high degree of symmetry between robot-to-human and human-to-robot approaches. However, these previous measurements were estimated from floor markings and were only accurate to within $10 \mathrm{~cm}$. The current study also found that approach distances for humans approaching the robot and robot approaching the human were comparable, though there were indications that humans approached the robot more closely than they allowed the robot to approach them by approximately 4 or $5 \mathrm{cms}$. This tentative finding will need confirming in further HRP studies with a larger number of participants.

Two participants left the study prematurely, stating that they were bored with the repetitive experimental procedures. This highlights issues related to the often incompatible demands of keeping experimental controlled conditions vs. having realistic, engaging and varied HRI trial scenarios. Sabanovic et al. [30] has argued that observational methods may usefully be applied to unstructured HRIs to gain more meaningful insights and findings than might be gained under relatively unnatural encounters found in highly controlled and restricted HRI trials. However, studies without clear structures and experimental protocols may suffer from inconclusive results due to factors that may or may not have had an influence during the study, which were not controlled for or measured. Thus, for long-term studies a careful balance has to be chosen between controlled and engaging experiments that enable users to maintain their interest in participating in multiple sessions.

Interestingly, the HRP preferences observed in this study provide an indication that participants statements, recollections and conscious perceptions of their encounters with robots often are at odds with how they actually respond! A similar discrepancy had been found in HRI studies by Kose-Bagci et al [31] whereby participants' perceptions of 'good human-robot interactions' were often inconsistent with their performance in human-robot drumming scenarios.

\section{CONCLUSION}

This study has overall confirmed HRP findings from previous studies. However, the enhanced HRP capabilities of the APS, in conjunction with the questionnaire and interview data has enabled a more comprehensive analyses of HRIs than previously possible. This has provided some, as yet tentative, indications of HRP behaviours being associated with participants unconscious sensitivities to the proxemic behaviour of the robot. This may well have implications for their perceptions of trust, comfort and in the robot. It is desirable for future work to carry out more comprehensive and focussed HRP studies into these aspects with larger numbers of participants in order to obtain a better basis for statistical analyses. 


\section{REFERENCES}

[1] E. T. Hall, "Proxemics." Current Anthropology, 1968, 9(23): pp. 83-108.

[2] E. T. Hall, Handbook for Proxemics Research. Society for the Anthropology of Visual Communication, Washington DC , 1974.

[3] R. Sommer, Personal Space: The Behavioral Basis of Design. Prentice Hall, New Jersey, 1969.

[4] J. K. Burgoon, S. B. Jones, "Toward a Theory of Personal Space Expectations and Their Violations." Human Communication Research, 1976, 2(2): pp. 131-146.

[5] C. Breazeal, B. Scassellati, "Infant-like Social Interactions Between a Robot and a Human Caretaker." Adaptive Behavior, 2000, 8(1): pp. 49-74.

[6] T. Nomura, T. Shintani, K. Fujii, K. Hokabe, "Experimental Investigations of Relationships Between Anxiety, Negative Attitudes, and Allowable Distance of Robots (HCI 07)" in Proceedings of the Second Interantioanal Conference on Human-Computer Interation (HCI 07). 2007. pp. 13-18

[7] H. Hüttenrauch, K. Severinson Eklundh, A. Green, E. A. Topp, "Investigating Spatial Relationships in Human-Robot Interaction" in Proceedings of the IEEE/RSJ International Conference on Intelligent Robots and Systems (IROS 06). 2006. pp. 5052-5059

[8] M. L. Walters, K. Dautenhahn, K. L. Koay, C. Kaouri, R. te Boekhorst, C. L. Nehaniv, I. Werry, D. Lee, "Close Encounters: Spatial Distances Between People and a Robot of Mechanistic Appearance" in Proceedings of IEEE-RAS International Conference on Humanoid Robots (Humanoids2005). 2005. pp. 450-455

[9] M. L. Walters, K. Dautenhahn, K. L. Koay, C. Kaouri, S. N. Woods, C. L. Nehaniv, R. te Boekhorst, D. Lee, I. Werry, "The Influence of Subjects' Personality Traits on Predicting Comfortable Human-Robot Approach Distances" in Proceedings of Cog Sci 2005 Workshop: Toward Social Mechanisms of Android Science. 2005. pp. 29-37

[10] M. L. Walters, K. Dautenhahn, S. N. Woods, K. L. Koay, R. te Boekhorst, D. Lee, Exploratory Studies on Social Spaces between Humans and a Mechanical-looking Robot. Journal of Connection Science, Special Issue on Android Science. 2006. pp. 429-442.

[11] M. L. Walters, K. L. Koay, K. Dautenhahn, R. te Boekhorst, D. S. Syrdal, "Human Approach Distances to a MechanicalLooking Robot with Different Robot Voice Styles" in Proceedings of the 17th IEEE International Workshop on Robot and Human Interactive Communication (RO-MAN 2008). 2008. pp. 707-712

[12] Koay, K. L., Syrdal, D. S., M. L. . Walters, K. Dautenhahn, "Living with Robots: Investigating the Habituation Effect in Participants' Preferences During a Longitudinal Human-Robot Interaction Study" in Proceedings of the 16th IEEE International Workshop on Robot and Human Interactive Communication (RO-MAN 2007). 2007. pp. 564-569

[13] J. T. Butler, A. Agah, "Psychological Effects of Behavior Patterns of a Mobile Personal Robot." Autonomous Robots, 2001, 10: pp. 185-202.

[14] D. S. Syrdal, K. L. Koay, M. L. Walters, K. Dautenhahn, "A Personalised Robot Companion? - The Role of Individual Differences on Spatial Preferences in HRI Scenarios" in Proceedings of the 16th IEEE International Workshop on Robot and Human Interactive Communication (RO-MAN 2007). 2007. pp. 26-29
[15] M. L. Walters, D. S. Syrdal, K. Dautenhahn, R. te Boekhorst, K. L. Koay, "Avoiding the Uncanny Valley - Robot Appearance, Personality and Consistency of Behavior in an Attention-Seeking Home Scenario for a Robot Companion." Journal of Autonomous Robots, 2008, 24(2): pp. 159-178.

[16] L. R. Goldberg, "The Structure of Phenotypic Personality Traits." American Psychologist, 1993, 48: pp. 26-34.

[17] T. Tamura, S. Yonemitsu, A. Itoh, D. Oikawa, A. Kawakmi, Y. Higashi, T. Fujiimooto, K. Nakajima, Is an Entertainment Robot Useful in the Care of Elderly People with Severe Dementia?. Gerontology. 2004. pp. 83-85.

[18] J. Mumm, B. Mutlu, "Human-Robot Proxemics: Physical and Psychological Distancing in Human-Robot Interaction" in Proceedings of the 6th ACM/IEEE International Conference on Human Robot Interaction (HRI'11). 2011. pp. 331-338

[19] Wakamuru. http://www.mhi.co.jp/en/products/detail/wakamaru.html.

[20] M. L. Walters, S. N. Woods, K. L. Koay, K. Dautenhahn, "Practical and Methodological Challenges in Designing and Conducting Human-Robot Interaction Studies" in Proceedings of the AISB'05 Symposium on Robot Companions Hard Problems and Open Challenges in Human-Robot Interaction. 2005. pp. 110-119

[21] M. A. Oskoei, M. L. Walters, K. Dautenhahn, "An Autonomous Proxemic System for a Mobile Companion Robot" in Proceedings of the Second International Symposium on New Frontiers in Human-Robot Interaction at the AISB 2010 Convention. 2010. pp. 9-15

[22] M. L. Walters, K. Dautenhahn, R. te Boekhorst, K. L. Koay, "An Empirical Framework for Human Robot Proximity" in Proceedings of Artificial Intelligence and Simulation of Behaviour Convention (AISB 09), New Frontiers in HRI Symposium . 2009. pp. 144-149

[23] A. Haasch, S. Hohenner, S. Hüwel, M. Kleinehagenbrock, S. Lang, I. Toptsis, G. A. Fink, J. Fritsch, B. Wrede, G. Sagerer, "BIRON - The Bielefeld Robot Companion" in Proceedings of the International Workshop on Advances in Service Robotics. 2004. pp. 27-32

[24] Y. Zheng, Y. Meng, Real-time People Tracking and Following Using a Vision-Controlled Mobile Robot. In Robot Vision: New Research. T. Matsuda (Ed.). 2009. pp. 149-160.

[25] H. Zender, P. Jensfelt, G. M. Kruijff, "Human- and Situation-Aware People Following" in Proceedings of the 16th IEEE International Workshop on Robot and Human Interactive Communication (RO-MAN 2007). 2007. pp. 1131 - 1136

[26] N. Bellotto, H. Hu, "Multisensor-Based Human Detection and Tracking for Mobile Service Robots." IEEE Transactions on Systems, Man, and Cybernetics, 2009, 39(1), Part B: pp. 167-181.

[27] E. T. Hall, The Hidden Dimension. Doubleday, NY, 1966.

[28] Mobile Robots. http://www.mobilerobots.com/.

[29] M. L. Walters, K. L. Koay, S. N. Woods, D. S. Syrdal, K. Dautenhahn, "Robot to Human Approaches: Comfortable Distances and Preferences" in Proceedings of the AAAI Spring Symposium on Multidisciplinary Collaboration for Socially Assistive Robotics, (AAAI SS07-2007). 2007.

[30] S. Sabanovic, M. P. Michalowski, R. Simmons, "Robots in the Wild: Observing Human-Robot Social Interaction Outside the Lab" in Proceedings of IEEE International Workshop on Advanced Motion Control (AMC 06). 2006. pp. 756-761

[31] H. Kose-Bagci, K. Dautenhahn, D. S. Syrdal, C. L. Nehaniv, "Drum-mate: Interaction Dynamics and Gestures in Human-Humanoid Drumming experiments." Connection Science, 2010, 22(2): pp. 103-134. 